\section{Ahmed PA Ulonnam CC}

\title{
Clinical presentation of tuberculosis in adolescents as seen at National Hospital Abuja, Nigeria
}

DOI:http://dx.doi.org/10.4314/njp.v41i4,8

Accepted: 3rd May 2014

Ahmed PA

Ulonnam CC $\square$ )

Department of Paediatrics,

National Hospital,

Abuja Nigeria.

Email: ahmedpatience@yahoo.com

\begin{abstract}
Background: Adolescents with tuberculosis (TB) form a significant proportion of childhood TB cases presenting with specifics clinical patterns.

Objective: To describe the clinical presentation of tuberculosis in adolescent at National Hospital Abuja (NHA), Nigeria.

Subjects and method: This is a descriptive and retrospective study of adolescents aged 10-15 years seen at the department of Paediatrics NHA Nigeria from August 2009 to July 2013.

Result: Thirty- six adolescents diagnosed with tuberculosis were reviewed. Adolescent TB accounted for $18.8 \%(36 / 192)$ of total cases of children aged 015years seen at the Department of Paediatrics Respiratory Clinic during the study period. The mean (SD) age was 12.3(1.76) years. Twenty seven patients (75.0\%) were females and $9(25.0 \%)$ were males. Thirty $(83.3 \%)$ were of lower socioeconomic class. History of contact with a case of TB was obtained in 17(47.2\%). The commonest symptoms identified in these adolescents were; cough $27(75.0 \%)$, weight loss 22 (61.1\%), fever18(50.0\%), sputum 14(38.9\%), body swelling 7(19.4\%), hemoptysis 2(5.6\%); while signs were underweight, pyrexia and chest findings. Nine (25.0\%) had associated retroviral disease. Thirty four $(94.4 \%)$ presented at time of diagnosis with
\end{abstract}

clinical symptoms while 2(5.6\%) were asymptomatic; identified during contact tracing as latent $\mathrm{TB}$ infection (LTBI). Abnormal chest radiograph findings were; widespread lung infiltrate in 10(27.8\%), hilar opacities $7(19.4 \%)$, cavitatory lesions $4(11.1 \%)$, pleural Effusion $3(8.3 \%)$ and military opacities 1(2.7\%). AFB was isolated in 5(13.9\%), while $23(63.9 \%)$ had a raised ESR above $30 \mathrm{~mm} / \mathrm{hr}$. Twenty seven $(75.0 \%)$ of the adolescents completed treatment for tuberculosis, $7(19.4 \%)$ were lost to follow up and $2(5.6 \%)$ died while $4(11.1 \%)$ had re-treatment for TB from relapse. Clinical presentations were pulmonary TB (PTB) 22(61.1\%), and extrapulmonary TB 12(33.3\%); distributed as TB adenitis 4(11.1\%), TBM 3(8.3\%), Pericardial TB 3(8.3\%), Miliary TB 1(2.8\%) and Spinal TB 1(2.8). Of the nine with HIV- TB coinfection, the clinical presentation were; PTB 5(55.6\%), and extrapulmonary 4(44.4\%).

Conclusion: The patterns of TB in adolescents are admixture as seen in younger children and adult from clinical and radiological characteristic findings. TB remains a preventable disease condition and is curable with early appropriate treatment

Key words: Adolescent tuberculosis, clinical pattern.

\section{Introduction}

The adolescent person whose age is between $10-19$ years, has been described generally to be a healthy group compared to the general population, despite their known high risk behavior ${ }^{1}$. The adolescent who is also a young person suffers from TB along with other mem- bers of the general population ${ }^{2,3}$. In 2012, WHO estimates that 8.6 million incident cases of TB were reported with 530,000 new case of TB being children less than 15 years and 74,000 child deaths from the disease ${ }^{2}$. These estimate were higher than the report published in the preceding year reflecting that more TB cases were being notified among children globally ${ }^{2}$. Over 30 
million Nigerians (approximately 22 percent) are between the ages of 10-19. ${ }^{4}$ The adolescents and Young people represent a high risk sexual behavior groups that are highly vulnerable to HIV /AIDs infection and consequently at risk of TB, in the dual pandemic ${ }^{5}$.

The younger child, under five have been shown to be at greater risk of TB especially in the presence of malnutrition and lower family socioeconomic background ${ }^{6,7}$. Childhood TB cases proportions in countries vary from 3 - 40percent ${ }^{2}$. Nigeria is among the 22 high burden nations with TB, accounting for 80percent of the world TB cases. An estimated 1.1 million (13percent) of the 8.6 million people who developed TB in 2012 were HIV positive with 75 percent in the African region ${ }^{2}$. This ancient and still ongoing TB scourge is caused by the mycobacterium tuberculosis complex, with high and low prevalence worldwide despite available measures that highlight how to addressed and control the disease. ${ }^{8}$

${ }^{9}$ The young person will be at risk of TB infection when in contact with a case of TB disease who is actively coughing, especially when he is immune compromised, is homeless or lives in congested camps, prison or jail, or nursing home and have risky behaviors such as intravenous drug use $\mathrm{e}^{2,3}$.

There are few reports that have focused on TB burden in the adolescent. In the United States, adolescents comprised approximately one-third of the pediatric cases reported from 1994 to $2010^{3}$. The younger child, particularly the under-fives play little role in the transmission of TB because they more often have negative smears; they rarely have cavitary disease; they often have little or no cough; and when cough is present, it is generally not forceful enough to expel aerosolized bacilli efficiently. ${ }^{10}$ In contrast, the 10 - to 19 -year age group presents a different spectrum of disease manifestations, including adult-type disease, from which respiratory samples can be more readily obtained ${ }^{2}$. The Stop TB Partnership goals include reducing the global burden of TB (prevalence and mortality) by 50 per cent in 2015 compared with 1990 levels and eliminating TB as a public health problem by $2050^{11}$. In other to achieve this goal, the adolescent though largely a subset of the paediatric age group but differ remarkably from the younger child should be viewed differently since preventive measure for TB differ for the child and the adult.

The study aims to describe the clinical presentation and outcome of adolescent tuberculosis at National Hospital Abuja (NHA), Nigeria.

\section{Subjects and Method}

This is a retrospective, descriptive study, conducted at the Paediatric Respiratory Clinic of National Hospital Abuja. The clinic is held once weekly with average attendance of 15 patients seen per week. The case folders of adolescents aged 10- 15 years seen at the clinic from August 2009 to July 2013 who were diagnosed with tuberculosis were retrieved.
The protocol for diagnosis of tuberculosis in the clinic were based on:

Bacteriological identification of mycobacterium tuberculosis complex by direct smear microscopy performed using auramine-rhodamine and confirmed with Kinyoun stain on clinical specimen in a patient with suggestive symptoms and signs or

Clinical diagnoses based on a combinationof symptoms and signs in the presence of any one or more of the following; a tuberculin skin test (TST) $\geq 10 \mathrm{~mm}$ for patients that are HIV negative or $\geq 5 \mathrm{~mm}$ in HIV positive patients, a history of contact with suspected tuberculosis patient, radiological and/ or histo-pathological finding from lymph node biopsy suggestive of TB. Such clinical features include a history of cough lasting for two weeks or more, sputum with or with haemoptysis, prolonged fever for which patient has been treated with antibiotics with no improvement, weight loss or malnutrition and poor appetite. Other symptoms include convulsions, loss of consciousness, swelling in the back or any other part of the body. Signs recorded were also retrieved including laboratory test results, treatment given with duration and outcome. Cultures were not done on any of the specimens. Only children aged less than 16 years were seen at the paediatric respiratory clinic as part of the hospital policy, while those 16 years and above were attended to by the adult physicians.

A proforma was used to extract information relating to patient demography, clinical symptoms and signs, history of contact, pattern of $\mathrm{TB}$, radiological features, treatment and outcome (completion of treatment, lost to follow up or retreatment for TB). All the patients were under care by the authors and case folders retrieved were analyzed after approval was obtained from the institutional review board of the National hospital Abuja. Data were analyzed using Microsoft excel 2010 and mean (SD), percentages and tables were generated.

\section{Results}

A total of 52 adolescents aged 0-15 years diagnosed with tuberculosis were documented in the paediatrics respiratory clinic record, but only 36 case folders with adequate information retrieved from the hospital medical records were analyzed during the period August 2009 to July 2013. This gives a prevalence rate of $18.8 \%$ (36/192). The mean (SD) age was $12.3(1.76)$. $75.0 \%$ of the adolescents were females while $83.3 \%$ were of lower socioeconomic group as shown in table 1.

\begin{tabular}{|c|c|c|c|}
\hline Iation & Variable & $\mathrm{N}$ & $\%$ \\
\hline & Sex & & \\
\hline & Male & 9 & 25.0 \\
\hline & Female & 27 & 75.0 \\
\hline & Socioeconomic stat & & \\
\hline & High social class & 6 & 16.7 \\
\hline & Lower social class & 30 & 83.3 \\
\hline
\end{tabular}


34 patients $(94.4 \%)$ were diagnosed after they presented with clinical symptoms while $2(5.6 \%)$ were identified through contact tracing as asymptomatic (latent TB cases). 17(47.2\%) adolescents had a history of contact with a person with suspected TB while $9(25.0 \%)$ had associated retroviral disease. Thirty two $(88.9 \%)$ had a history of BCG given within one month of life. The most common symptoms identified were cough, weight loss and fever, while major signs were underweight, pyrexia and chest findings (respiratory distress, tachpnoea, crepitations, decreased percussion note and chest expansion); table 2.

Acid- alcohol fast bacillus (AFB) was isolated in 5 $(13.9 \%)$ and $23(63.9 \%)$ had a raised ESR above $30 \mathrm{~mm} /$ $\mathrm{hr}$, Abnormal chest radiograph findings were; widespread lung infiltrate $10(27.8 \%)$, hilar opacities 7(19.4\%), cavitory lesions $4(11.1 \%)$, pleural effusion $3(8.3 \%)$ and miliary opacities $1(2.8 \%)$. Twenty seven (75.0\%) of the adolescents completed treatment for tuberculosis, 7(19.4\%) were lost to follow up and $2(5.6 \%)$ died., while $4(11.1 \%)$ had re-treatment for TB due to relapse.

\begin{tabular}{lcc}
\hline $\begin{array}{l}\text { Table 2: Common symptoms and signs identified in adoles- } \\
\text { cent TB }\end{array}$ & N & $\%$ \\
Symptoms & 27 & 75.0 \\
\hline Cough & 22 & 61.1 \\
Weight loss & 18 & 50.0 \\
Fever & 14 & 38.9 \\
Sputum & 7 & 19.4 \\
Body Swelling (various sites) & 3 & 8.3 \\
Headache & 2 & 5.6 \\
Hemoptysis & 1 & 2.8 \\
Swelling in the back & & \\
Signs & 24 & 66.7 \\
Underweight $\leq 80 \%$ & 17 & 47.2 \\
Pyrexia & 16 & 44.4 \\
Pallor & 15 & 41.7 \\
Chest findings & 11 & 30.6 \\
Hepatomegaly & 7 & 19.4 \\
Lymphadenopathy & 2 & 5.6 \\
Gibbus (thoracolumbar) & 1 & 2.8 \\
Neck stiffness & &
\end{tabular}

The clinical presentations of TB were; \%) pulmonary TB (PTB) 22(61.1) and extra-pulmonary TB 14 (38.9\%); table 3

\begin{tabular}{lll}
\hline \multicolumn{3}{c}{ Table 3: Clinical presentation of TB in the adolescents } \\
Variable & $\mathrm{N}$ & $\%$ \\
\hline Latent TB & 2 & 5.6 \\
Pulmonary TB & 22 & 61.1 \\
Smear positive & 5 & 13.9 \\
Smear negative & 17 & 47.2 \\
Extrapulmonary TB & 12 & 38.9 \\
TB adenitis & 4 & 11.1 \\
TB meningitis & 3 & 8.3 \\
TB pericarditis & 3 & 8.3 \\
Tb spinal & 1 & 2.8 \\
Milary TB & 1 & 2.8 \\
\hline
\end{tabular}

Of the nine cases with HIV-TB co- infection, 5(55.6\%) presented pulmonary disease while remaining 4 had extra- pulmonary diseases; namely TBM 2; pericardial $\mathrm{TB}$ and miliary TB one each; in Chart 1
Chart 1: TB pattern in adolescent with retroviral disease

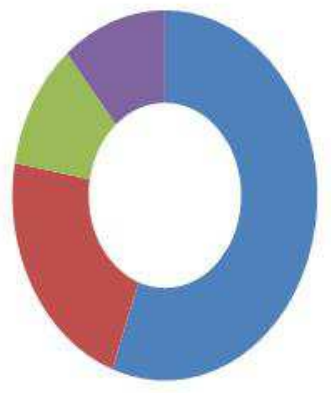

TBM $=2$ CASES

In PERICARDIAL TB $=1$ CASE

MILIARYTB $=1$ CASE

\section{Discussion}

The study describes the clinical characteristics of adolescent TB at the National Hospital Abuja. Adolescent TB accounted for 18.8 percent of cases of TB seen during the study period. The female was at greater risk of TB in this report. Studies have documented that the prevalence of TB in children is higher in girl until between the age 10 and 16 years $^{12}$. The gender disparity becomes evidence after fifteen years with a rising rate amongst male up till adulthood ${ }^{12}$. Majority of the cases had active disease. This may be the reason why the prevalence was higher in the female since the progression from infection to active disease is more rapid in the female gender early in life and the reverse at older age $\mathrm{e}^{12,13}$. The young girl has been shown to have a threefold higher to acquire HIV infection when compared to the male of same age. This risk factor for HIV may also contribute to early active disease progression ${ }^{10}$. Other factors that have been known to account for considerable variability in the outcome of M. tuberculosis infection include malnutrition, poor socioeconomic status and immune suppression such as caused by $\mathrm{HIV}^{4,5,14-16}$. Over 60 percent of the study populations were undernourished with weights 80 percent or less for age and sex. Malnutrition interferes with the cell mediated immunity (CMI) response and therefore contributes to much of the increased frequency of TB in impoverished patients ${ }^{16}$. The TB rates were highest in the adolescent of lower socioeconomic status. The historical association of TB with low socioeconomic status and poverty had long been established ${ }^{14,15}$. HIV/AIDS-TB co- infection coexisted in twenty five percent of the adolescents. The dual disease burdens are known to adversely affect the outcome of each other. HIV impairs the host cellular immunity to contain TB infection and verse visa. The combination with HIV co-infection, dramatically compromises host resistance to $\mathrm{TB}$, leading to high disease prevalence in affected endemic populations that include the adolescent. With the current over half a million adolescent infected with TB as at $2012,{ }^{2}$ the risk for increasing prevalence of multidrug-resistant (MDR) and extensively drug-resistant (XDR) MTB strains ${ }^{17}$ and the more recent occurrence of TDR (totally drug-resistant) MTB strains, which are virtually untreatable ${ }^{18}$ becomes a challenge to all for early detection and treatment of all cases of TB. 
Evidence of late presentation in 94.4 percent of the adolescents studied was the presence of clinical symptoms at time of diagnosis. Such adolescents' patients would have already contributed to infection transmission especially as they cough with sputum production in the presence of AFP positive status acting as reservoir of infection to contacts. Late diagnosis results from this lack of early clinical suspicion of the disease. Cough was the commonest symptom in the adolescent while 38.9 perecnt were able to produce sputum, unlike the younger child who most times cannot expectorate voluntarily. Cough as a significant symptom was also found to be specific in a report by Marais et $\mathrm{al}^{19}$ in which a persistent, non-remitting cough was reported in $15 / 16$ $(93.8 \%)$ children under 13years with tuberculosis and in 2/135 (1.5\%) children and young adolescents, without tuberculosis, indicating a specificity of $98.5 \%(135 / 137)$ 19. The use of well-defined symptoms, even in resource limited settings offer some value in the diagnosis of childhood and adolescent pulmonary tuberculosis. Sputum production with acid-alcohol fast bacillus (AFB) smear positivity is risk factors for transmission of infection among the adolescents and among his peers due to the high social interaction associated with this age group. Close to 14 percent were bacteriological confirmed cases of TB in the present report. Contact with person with suspected TB is an important factor for acquiring the infection seen in up to 47.2 percent of the adolescents. Early case finding from screening of contacts of adolescents and adult cases of active cases remains a strategy for the prevention and control of TB. This also includes the use of isonaizid prophylaxis especially in under five children exposed to contacts of active disease and HIV positive persons. All adolescents with a contact history must be evaluated with a TST for LTBI. Two cases were found to have LTBI in this review and treated. The practice of LTBI treatment for TB contacts with a TST size of $10 \mathrm{~mm}$ and above help reduce progression to disease. This routine use of isoniazid preventive therapy (IPT) is an effective and costeffective intervention among child household contacts.

The use of Bacillus Chalmette -Guerin (BCG) vaccine was high among the adolescents. The BCG vaccine have been shown to have from zero to 80 percent effectiveness in preventing tuberculosis for a duration of 15 years; however, its protective effect appears to vary according to geography and the laboratory in which the vaccine strain was grown ${ }^{20,21}$. A 1994 systematic review found that the BCG reduces the risk of getting TB by about $50 \%$; (relative risk (RR) of TB of 0.49 (95\% confidence interval [CI], 0.34 to 0.70 ) for vaccine recipients compared with non-recipients (protective effect of 51\%), and a protective effect from tuberculous deaths of $71 \%$ (RR, $0.29 ; 95 \% \mathrm{CI}, 0.16$ to 0.53 ), and a protective effect for meningitis of $64 \%$ (OR, 0.36 ; $95 \% \mathrm{CI}, 0.18$ to $0.70)^{22}$. BCG is effective against rare forms of severe childhood TB meningitis and miliary disease, however, the variation in protection against common pulmonary TB that BCG offers has generally been disappointing in trials conducted in the developing world ${ }^{23,24}$. This protection of BCG for children from severe forms of TB in the first years of life, however doesn't prevent pulmonary $\mathrm{TB}$, which is prevalent in the adolescents and adults, who mostly spread the infection. A BCG that will prevent progression of latent $\mathrm{TB}$ to active disease would be desirable; hence a vaccine that is more effective and safe against TB remains a task. The possible role of a booster or replacement in the ongoing BCG trials may in future provide the solution to TB infection in the population that includes adolescents ${ }^{25}$.

The patterns of TB in adolescents seen are a mixture of childhood forms and adult patterns in their clinical, radiological and microbiological findings. Most of the childhood TB forms seen were smear negative PTB similar to the finding reported by Cruz AT et al. ${ }^{3}$ For the extra- pulmonary forms, hilar lymphadenopathy was commonest followed by tuberculous meningitis and pericarditis occurring in equal proportion. A similar pattern was also identified in the adolescent with retroviral disease.

Imaging studies such as chest radiograph plays a significant role in childhood TB diagnosis. A normal radiographic study does not however rule out $\mathrm{TB}^{26}$. Abnormal chest radiograph findings were mostly widespread lung infiltrate (27.8 percent), hilar opacities (19.4percent), cavitatory lesions (11.1percent), pleural effusion (8.3percent) and miliary opacities (2.7percent). Traditionally, the two radiological patterns of TB manifestations that have been described are the primary TB and reactivation (post-primary) TB which relate to the patient immunity ${ }^{27}$. The present study shows a mixed form of both primary disease and reactivation $\mathrm{TB}$, though primary pattern was more predominant. Children and the immunocompromised persons usually present with features of primary TB which includes; parenchymal infiltrates or consolidation within the lung parenchyma, involving any pulmonary lobe or segment, hilar lymph node with or without atelectasis and unilateral pleural effusion on the same side of the primary focus of the $\mathrm{TB}^{28}$. In Reactivation TB, apical consolidations involving the upper lobe or superior segment of lower lobe are commonly seen with other features of cavitation, solitary nodules and military picture min some cases ${ }^{28}$. The primary forms of TB were mostly those of hilar lymph node enlargement, (52.8 percent). Marias et $\mathrm{al}^{29}$ reported that up to $90-95$ percent of primary $\mathrm{Tb}$ in children are hilar lymph node enlargement which could be unilateral or bilateral and widespread in up to 70 percent.

Completion of treatment in TB is a significant part of disease control, as this stops transmission of infection from an active case to contacts. A 75percent complete treatment rate in the present report was slightly lower than the reported 85percent national figure (Nigeria $2009-2013)^{30}$. A loss to follow up or default from treatment and poor compliance are risk factors for continuing infection transmission in the community with risk for resistance development and drug resistance TB. Lost to follow up rate was 19.4percent; which is lower than earlier report by Jiya et al $(36.5 \%)$ from Sokoto in northwest of Nigeria ${ }^{5}$. The Directly Observed Therapy (DOT) 
strategy aim to improve treatment compliance and completion for the patients through a reliable supervisor of patients and accurate record keeping. TB treatment in the adolescent can be a challenge resulting from the diverse behavioral, social and economic demography associated with the development of the adolescent. This can lead to treatment discontinuation, resulting in perpetuation of TB transmission in the community and appearance of resistant strains. Death rate among the adolescent was 5.6 percent; similar to the overall TB deaths of five percent for 2010 (Nigeria) ${ }^{31}$.

\section{Conclusion}

Tuberculosis remains a significant public health problem, particularly in resource limited settings like Nigeria. Late presentation was high in majority of adolescent as they were symptomatic already before presentation for diagnosis. A mixed pattern of disease was seen in the adolescent for a disease condition that remains preventable and treatable.

\section{References}

1. World Health Organization, online article; Adolescent health. Link: http://www.who.int/topics/ adolescent_health/en/ (Accessed: 26/10/2013)

2. World Health Organization, Geneva. Global Tuberculosis Report 2013.Link:http://apps.who.int/iris/ bitstream/10665/91355/1/ 9789241564656_eng.pdf?ua=1 (Accessed 26/10/2013)

3. Cruz AT, Hwang KM, Birnbaum GD, Starke JR. Adolescents with Tuberculosis. A Review of 145 Cases. Pediatr Infect Dis J. 2013;32(9):937-941

4. Esiet AO. Action Health Inc. Adolescent Sexual and Reproductive Health in Nigeria. Why "Adolescent Sexual and Reproductive Health". Link: http:// www.wilsoncenter.org/sites/ d e fault/files / E s i e t \% 20Presentation.pdf (Accessed 20/11/2013)

5. World Health Organanization. online article: Nigeria- link: www.who.int/countries/nga/en/ (Accessed: 27/11/2013)

6. Ibadin MO, Oviawe O. Trends in childhood tuberculosis in Benin City, Nigeria. Ann Trop Paed 2001; 21:141-145

7. Jiya NM, Bolajoko TA, Airede KI. Pattern Of Childhood Tuberculosis In Sokoto, Northwestern Nigeria. Sahel Medical Journal 2008;11 (4):110-113

8. Wu $\mathrm{XR}$, Yin QQ, Jiao $\mathrm{AX}, \mathrm{Xu}$ PB, Sun L, Jiao WW, et al. "Pediatric Tuberculosis at Beijing Children's Hospital: 2002-2010," Paediatrics,2012; 130(6):14331440.

9. Winston C, Menzies H. Pediatric and adolescent tuberculosis in the United States, 2008-2010. Pediatrics. 2012;130(6) 1425- 1432

10. Lawrence RM. Tuberculosis in children. In: Rom WN, Garay SM, eds. Tuberculosis. Boston: Little, Brown, 1996:675-88.
11. World Health Organization 2011. The global plan to stop TB 20112015. http://www.stoptb.org/ assets/documents/global/plan/ TB_GlobalPlanToStopTB2011 2015.pdf.

12. World Health Organanization. Online article: Gender and Tuberculosis. Link: whqlibdoc.who.int/ gender/2002/a85584.pdf (Accessed 12/12/2013)

13. Hudelson P. Gender differentials in tuberculosis: the role of socioeconomic and cultural factors. Tubercle and Lung Disease 1996;77:391e400.

14. Gustafson P, Lisse I, Gomes V, Vieira CS, Lienhardt C, Naucler A, Jensen H, Aaby P. Risk factors for positive tuberculin skin test in Guinea-Bissau. Epidemiology. 2007; 18:340-7.

15. Grigg ER. The arcana of tuberculosis with a brief epidemiologic history of the disease in the USA. Am Rev Tuberc. 1958;78:151-72.

16. Cegielski JP, McMurray DN.The relationship between malnutrition and tuberculosis: evidence from studies in humans and experimental animals.Int J Tuberc Lung Dis. 2004 (3):286-98.

17. Gandhi NR, Nunn P, Dheda K, Schaaf HS, Zignol M, et al. Multidrug-resistant and extensively drug-resistant tuberculosis: a threat to global control of tuberculosis. Lancet 2010; 375: 1830-1843.

18. Velayati AA, Masjedi MR, Farnia $\mathrm{P}$, Tabarsi P, Ghanavi J, et al. Emergence of new forms of totally drug-resistant tuberculosis bacilli: super extensively drug-resistant tuberculosis or totally drugresistant strains in iran. Chest 2009; 136: 420-425.

19. Marais B, Gie R, Obihara C, Hesseling A, Schaaf H, and Beyers N. Well defined symptoms are of value in the diagnosis of childhood pulmonary tuberculosis. Arch Dis Child. 2005; 90(11): 1162-1165.
20. Sterne J. A., Rodrigues L. C., Guedes I. N. Does the efficacy of BCG decline with time since vaccination? Int. J. Tuberc. Lung Dis. 1998; (2) 200-207.

21. Ottenhoff TH, Kaufmann SH. Vaccines against tuberculosis: where are we and where do we need to go? PLoSPathog. 2012; 8(5), 1002607

22. Colditz GA, Brewer TF, Berkey CS, Wilson ME, Burdick E, Fineberg HV, Mosteller F. Efficacy of BCG vaccine in the prevention of tuberculosis. Meta-analysis of the published literature. JAMA 1994 ; 271(9):698-702

23. Fifteen year follow up of trial of BCG vaccines in south India for tuberculosis prevention. Tuberculosis Research Centre (ICMR), Chennai. Indian J. Med. Res. 1999; 110: 56-69

24. Rodrigues L. C., Diwan V. K., Wheeler J. G. Protective effect of BCG against tuberculous meningitis and miliary tuberculosis: a meta -analysis. Int. J. Epidemiol. 1993; 22:1154-1158

25. McShane H. Tuberculosis vaccines: Beyond BacilleCalmetteGuérin. Philos Trans R SocLond B Biol Sci. 2011; 366(1579): 27822789.

26. Lamont AC, Cremin BJ, Pelteret RM. Radiological pattern of Pulmonary tuberculosis in the paediatric age group. Pediatr Radiol 1986; 16: 2-7.

27. Awil PO, Bowlin SJ, Daniel TM. Radiology of pulmonary tuberculosis and HIV infection in Gulu, Uganda. EurRespr J.1997; 10: 615 $-618$

28. Ogbeide O. The radiological management of tuberculosis. Benin journal of postgraduate medicine. December 2009; 11: 74 - 78. 
29. Marais BJ, Gie RP, Schaaf HS, Hesseling AC, Enarson DA, Beyers N. The spectrum of childhood tuberculosis in a highly endemic area. Int J Tuberc Lung Dis 2006; 10:732-738.
30. World health Organization online article. Global Health Observatory (GHO).How many TB cases have been successfully treated? Link:http://www.who.int/gho/tb/ epidemic/treatment/en/. (Accessed 10/1/2014).
31. Nigeria Tuberculosis Fact Sheet. United States Embassy in Nigeria. Link:http://photos.state.gov/ libraries/nigeria/487468/pdfs/ JanuaryTuberculosisFactSheet.pdf. 2012. (Accessed 10/1/2014). 